\title{
The increasing relevance of public health research in the last 95 years
}

\author{
Michael Foth
}

Published online: 27 March 2012

(C) Springer-Verlag 2012

The first School of Public Health (John Hopkins School of Hygiene and Public Health in Baltimore 1916) was founded over 95 years ago. Public health was defined in 1920 by Winslow as the science and art of preventing disease, prolonging life, and promoting physical health and efficiency through organized community efforts. Since then, research in the area of health maintenance and disease prevention has become increasingly important (Gutzwiller 1993). Topics falling under the notion of Public Health are now seen in the analysis, evaluation and organization of the causes, developments and counter measures that affect either the health of a population or large sections of a population (Schwartz et al 1991).

The Journal of Public Health has set itself the task of exploring the developments, trends and results of this research by including a wide range of topics from global strategies and organizational requirements to medical research and development.

This issue of the Journal of Public Health presents articles within different fields in public health research, with a special focus on the organization of public health, organizational themes of hospitals, influences on employees working in hospitals and research papers in the area of public health medicine.

The use of graphical illustrations by public health researchers in scientific presentations was analyzed by Frederico et al. and concluded that the ability of public health researchers to fully exploit the visual potential of graphs of their findings appeared limited. The majority of

\section{Foth $(\bowtie)$}

Department of Public Health and Health Technology Assessment, UMIT - The Health \& Life Sciences University,

Eduard Wallnöfer-Zentrum 1,

6060 Hall in Tyrol, Austria

e-mail: michael.foth@umit.at the graphs were bar diagrams and linear diagrams in univariate displays. Graphics used in oral presentations were notable for clarity and use of colour.

Hemken et al. found that optional deductibles offered by German health insurance and their respective size had an effect, in the medium term (3 years), on the number of claims filed for health benefits. The study analyzed the volumes of claims in 2009, between groups with and without deductibles. From the group with deductibles, the relationship between the amounts claimed and the chosen size of the deductible was investigated. The results show significantly different costs for hospital treatment, drugs and remedies between the two groups, where claims submitted by those with deductibles were on average 707 Euros lower for the comparable health services.

The level of implementation of the Primary Health Care Master Plan in Unai (Brazil) evaluated by Arantes and Shimizu is in an initial stage. The Kruskal-Wallis test showed significant differences in the degree of implementation of Family Health Teams and to evaluate Primary Health Care structures, processes and outcomes. To achieve the optimal stage of its implementation the inconsistencies and gaps in the structure resources must be resolved before the processes and expected outcomes can be analyzed. The primary focus is still on women's and children's health, although the master plan is based on some principles from the WHO Declaration of Alma-Ata (World Health Organization 1978).

In China $\mathbf{X u}$ et al. explored the attitude of farmers toward the New Rural Cooperative Medical Scheme one year after implementation. Most of the 1,978 farmers hold a positive attitude towards the new medical scheme, but the farmers trust the village doctors more than they trust the scheme management and village cadres. Two-thirds of the 
farmers clearly prefer the county hospitals to the village and township hospitals. The results proposed a number of remedial actions with respect to health financing, planning and management to counteract the identified issues.

The management, characteristics and generation of healthcare waste in hospitals in Kampala (Uganda) and the assessment options are elements in the research work of Mugambe et al.. The rate of generation of infectious waste and general waste in one public and one private hospital is examined. The examination of the factors which influence the generation of waste can help the health facility managers to minimize the quantities of healthcare waste. No differences were found that suggest that either the public or private ownership hospital was more successful in the management of healthcare waste, but health-care-waste management services provided by a third-party (external contractor), as in public hospitals, had resulted in improved services and should also be tried in private hospitals.

Data-protection in the public health context is always a topic in the media when misuse of patient data by hospital employees occurs. Data-protection regulations based on data-protection laws and internal regulations must be complied with and taken into account by hospital employees in daily work. The influencing factors of hospital employees' on data-protection compliance in German hospitals was analyzed in a study by Foth et al. Significant differences related to the acceptance of data-protection rules and regulations were found by gender or hospital type. The results show that employees consider rules and regulations to be necessary and important. However damage caused by data security breaches and the likelihood that they will occur, are considered to be less significant.

Possible predictors of consuming filtered water in rural Ethiopia (due to the risk of high fluoride concentrations in the ground and surface water) derived from various behavior change theories, were examined by Huber et al. in a cross-sectional study. In four areas fluoride removal household filters based on bone char media have been implemented. The consumption of fluoride-free water is mainly related to an individual's pride in offering filtered water to guests and the feeling of being able to produce enough filtered water. The majority of the filter users like the taste of filtered water and the more expensive they perceive the filter media, the more users exclusively consume filtered water. The results could be a base for designing intervention strategies to increase the consumption of treated water by exploiting psychological factors.

Sundmacher et al. examined in a study the spatial distribution of avoidable cancer deaths in Germany by identifying small areas with exceptionally high cancer rates using districts as a unit of analysis. The results suggest that the highest avoidable cancer deaths rates seen for women and men under 70 years of age differ by type of cancer (for women cancer of the breast, trachea, bronchus and lung; for men cancer of the trachea, bronchus, lung, colon, rectosigmoid junction, rectum, anus and anal canal). The results show that the spatial distribution of avoidable cancer mortality rates in women in Germany showed a north-south gradient. When applying measures, the results suggest the use of areas with statistically lower avoidable cancer mortality rates than the German average as a benchmark for the maximum number of excess deaths that should be considered preventable.

The existing lack of data, outside the context of clinical trials, on the management and long-term outcomes of patients with primary or secondary antibody deficiency, or those with neurological autoimmune diseases treated with immunoglobulins was examined in the study of Kirch et al. using observations from the SIGNS registry. The SIGNS registry is based on a study of 550 patients in 50 sites throughout Germany. It investigates treatment patterns, effectiveness, quality of life and long-term outcomes of immunoglobulins administered for primary or secondary antibody deficiency or autoimmune diseases. Based on the broad spectrum of indications and immunoglobulins preparations documented in this registry, the registry will contribute to the optimization of therapy in this diverse patient population.

The researchers Kadayat et al. investigate the patterns and perception of complementary and alternative medicine use by patients in western Nepal. The study, in 52 clinics with 501 patients, shows that the use of complementary and alternative medicine is more common among patients with chronic conditions for which they had already tried allopathic medicine and that most of the patients viewed complementary and alternative medicine as beneficial, cheaper, and free of side effects.

A review seeking to identify effective asthma education programs geared toward educating pre- and adolescent youth in asthma management techniques was done by Alison Chrisler. Randomized controlled studies with focus on asthma education and disease management were examined for this review. The literature review indicated that the majority of the asthma programs were school or clinic-based and that the asthma education programs demonstrate favorable outcomes. The majority of the programs involved parents and some programs provided a group session specifically for parents. The results suggest that more asthma education programs that primarily use a strengths model, as seen in the web-based self-training program, and which also focus on the specific needs of youth need to be developed.

The review of Gerber et al. presents a systematic summary of studies examining the relationship between acculturation and physical activity among immigrants and ethnic minority populations. 44 original studies with 760,242 participants were reviewed which incorporated a 
measure of acculturation and physical activity, exercise, or sport. The results show that acculturation was associated with higher leisure time physical activity in $57 \%$ of all studies, independent of participant's gender, age and ethnic background. Prevention programs should give priority to individuals with low acculturation levels and it should consider culturally specific beliefs and constraints. Additionally, prevention programs could make an effort to promote acculturation among immigrants.

In a comment, Jayaram Srinivasan wrote about the meta-leadership in the implementation of the Farm to School model for improving childhood nutrition in Kentucky. Meta-leadership provided the foundation to help reduce childhood obesity and to help stimulate the local economy. The ability of the leader to successfully utilized innovative paradigms to mobilize various community partners and to leverage the resources of several organizations across public and private sectors are the substantial factors of this project.
Together, the articles in this issue of the Journal of Public Health illustrate very well the scope and reach of public health today. The results and findings of the contributions support the reader in further consideration of the challenges for current and future research in public health.

\section{References}

Gutzwiller F (1993) Gesundheitswissenschaften (Public Health): Eine interdisziplinaere Aufgabe. Sozial- und Praeventivmedizin 38:1, pp 1-7, Birkhaeuser

Schwartz F, Badura B, Brecht JG, Hofmann W, Jöckel KH, Trojan A (eds) (1991) Public health - Texte zu Stand und Perspektiven der Forschung. Springer, Berlin

Winslow C-EA (1920) The untilled fields of public health. Science $51: 23$

World Health Organization (1978) Declaration of Alma-Ata. http://www.who.int/hpr/NPH/docs/declaration_almaata.pdf. Accessed 20 February 2012 